\title{
UWB Signals as Shot Noises
}

\author{
A. Ridolfi \\ School of Computer and Communication Sciences \\ Ecole Polytechnique Fédérale de Lausanne \\ 1015 Lausanne, Switzerland \\ e-mail: andrea.ridolfi@epfl.ch
}

\author{
M. Z. Win \\ Laboratory for Information and Decision Systems \\ Massachusetts Institute of Technology \\ Cambridge, MA 02139-4307, USA \\ e-mail: moewin@mit.edu
}

\begin{abstract}
We present a shot-noise based model for a large family of ultrawide bandwidth (UWB) signals. These include time-hopping and directsequence signaling with pulse position, interval and amplitude modulations. Each specific signal is constructed by adding features to a basic model in a modular, simple, and tractable way. Our work unifies the contributions scattered in the literature and provides a general approach that allows various extensions of previous works. The exact power spectrum is then evaluated using shot-noise spectral theory, which provides a simpler, systematic, and rigorous approach to the spectra evaluation of complex UWB signals. The strength of our methodology is that different features of the model contribute clearly and separately in the resulting spectral expressions.
\end{abstract}

\section{INTRODUCTION}

UWB radio communicates with pulses of very short duration, thereby spreading the energy of the radio signal over several $\mathrm{GHz}$. UWB signals are transmitted with spread spectral content while maintaining the average power level required for reliable communications. As a consequence, the spectral characteristics (spectral occupancy and composition) of an UWB transmission has a key role in the design of UWB systems.

Exact spectral evaluation has already received attention in the communications community. Among several contributions, we mention the computation of the power spectrum of a general time-hopping, pulse position modulated signal [1]-[4], and that of the spectral density of the family of pulse interval modulated signals [5]. In these contributions, the signal models are based on Dirac pseudo-functions and lack generality, especially with respect to the type of temporal modulation. The spectrum computation is performed using the classical wide-sense stationary (w.s.s.) approach, i.e., as the Fourier transform of the correlation function. Although this is a common approach, the resulting computations are complex and the introduction of additional random features of the model, such as clock jitter, pulse losses or pulse distortion, requires a new computation from scratch.

Here we show that UWB signals are aptly modelled as shot noises with random excitation, i.e., a filtered stream of spikes at random times $\left\{T_{n}\right\}_{n \in \mathbb{Z}}$, where the filtering function $h(t, Z)$ depends on a random parameter $Z$

$$
X(t)=\sum_{n \in \mathbb{Z}} h\left(t-T_{n}, Z_{n}\right), \quad t \in \mathbb{R} .
$$

More precisely, the sequence of random times $\left\{T_{n}\right\}_{n \in \mathbb{Z}}$ determines the temporal structure of the signal and the random function $h(t, Z)$ characterizes the shape of the pulses and its random modifications (amplitude, displacement, distortion). Shotnoise processes have received much attention in the applied literature (see for instance [6], [7] and the references therein). Concerning communications systems, they have been widely used in queuing and teletraffic theory [8], and a Poisson based model for pulse coded optical transmissions has been proposed in [9], [10]. In the context of UWB signals, a Poisson shot-noise was proposed by [11] to model interference on narrowband systems.

As we shall see, there are two considerable advantages in modeling UWB signals as shot-noise processes. Firstly, the model is modular, simple, and tractable. Thus, one can construct different UWB signals in a unifying way by simply adding features to a basic model systematically, and can easily take into account random quantities, such as jitter, losses, or pulse distortions, which affect the signals. Secondly, spectra are obtained, in a systematic and rigorous manner from a single general formula. This formula simplifies previous proofs of the existing results and provides spectrum expressions of highly complicated signals where various features of the model appear 
separately and explicitly, preserving the modularity of the model. As we will see, these advantages have a tremendous impact on the design and the analysis of UWB signal models.

UWB transmission systems employ time-hopping or direct-sequence signals to achieve multiple access with pulse position or pulse amplitude modulation for data transmission (see for instance [1], [12]). Here, we focus on the following schemes: pulse amplitude modulation (PAM), pulse position modulation (PPM), time-hopping ( $\mathrm{TH})$ signals and directsequence (DS) signals. However, our approach can be easily extended to other signals such as (digital) pulse interval modulation (DPIM), (digital) pulse interval and amplitude modulation (DPIAM), and to any combination of pulse modulations and multiple access techniques (see [13]).

\section{Shot Noise Models of Uwb Signals}

We now give some examples of shot noise modeling of UWB signals. In particular we focus on pulse position and amplitude modulation, time-hopping pulse position and amplitude modulation and directsequence time-hopping pulse position and amplitude modulation, all cases in the presence of clock jitter and random losses.

\section{A. Pulse Position and Amplitude Modulated Signals}

In pulse position modulation (PPM) the information is carried by the relative distance of the pulses with respect to a regular grid (see for instance [1], [12], [14]-[16]). Therefore, a PPM signal can be seen as a regularly spaced stream of spikes convoluted with the pulse shape, to which we add positive random i.i.d. displacements coding the symbols to be transmitted. The points of $N$ are given by

$$
T_{n}=U+n T, \quad n \in \mathbb{N},
$$

where $U$ is a uniform- $[0, T]$ random variable, that ensures the stationarity of the random times $\left\{T_{n}\right\}_{n \in \mathbb{Z}}$, and $T$ is the period of repetition of the spikes. The random displacements, coding the symbols, are modelled using the random parameters $\left\{Z_{n}\right\}_{n \in \mathbb{Z}}$. Calling $w(t), t \in \mathbb{R}$, the pulse shape, a PPM signal is modelled as

$$
X(t)=\sum_{n \in \mathbb{Z}} w\left(t-U-n T-Z_{n}\right),
$$

Hence, we have the shot noise with random excitation of equation (1), where $T_{n}:=U+n T$, and $h(t, Z):=$ $w(t-Z)$.
In the presence of i.i.d. jitter and i.i.d. random losses, the pulse position modulated signal reads

$$
X(t)=\sum_{n \in \mathbb{Z}} Z_{n}^{\mathrm{L}} w\left(t-U-n T-Z_{n}^{\mathrm{P}}-Z_{n}^{\mathrm{J}}\right),
$$

where $\left\{Z_{n}^{\mathrm{L}}\right\}_{n \in \mathbb{Z}}$ is the sequence of $\{0,1\}$-valued random variables modeling the random losses, $\left\{Z_{n}^{\mathrm{P}}\right\}_{n \in \mathbb{Z}}$ is the sequence of random variables modeling the position modulation and $\left\{Z_{n}^{J}\right\}_{n \in \mathbb{Z}}$ is the sequence modeling the additional random displacements due to the clock jitter. Therefore, we have the shot noise of equation (1) with with filtering function

$$
h(t, Z):=Z^{\mathrm{L}} w\left(t-Z^{\mathrm{P}}-Z^{\mathrm{J}}\right),
$$

i.e., the random parameters are now the vectors $Z_{n}:=\left(Z_{n}^{\mathrm{L}}, Z_{n}^{\mathrm{P}}, Z_{n}^{\mathrm{J}}\right), n \in \mathbb{Z}$.

We can easily add the amplitude modulation, by now considering the model (1) with random impulse function

$$
h(t, Z):=Z^{\mathrm{A}} Z^{\mathrm{L}} w\left(t-Z^{\mathrm{P}}-Z^{\mathrm{J}}\right),
$$

i.e., we add to the random vector $\left\{Z_{n}\right\}_{n \in \mathbb{Z}}$ the sequence $Z_{n \in \mathbb{Z}}^{\mathrm{A}}$ modeling the i.i.d. random amplitudes of the pulses.

The case of correlated random amplitudes can also be easily taken into account [13], [17].

\section{B. Time-Hopping Pulse Position and Amplitude Modulated Signals}

General time-hopping ( $\mathrm{TH})$ signals are characterized by a deterministic periodic pattern (or pseudorandom) that allows for multi-user detection. Such a pattern, commonly called signature, consists of a sequence of pulses positioned with respect to a regular grid.

The temporal structure of such a signal is described by the random times

$$
T_{n}+\sum_{l=0}^{L_{c}-1}\left(l T+c_{l} T_{c}\right),
$$

where

- $\left\{c_{n}\right\}, n=0, \ldots, L_{c}-1$, is the deterministic sequence characterizing the $L_{c}$-periodic pattern;

- $T$ is the period of the regular grid $\left(T_{c}<T\right)$;

- $T_{n}$ is a sequence of random times that depends on the type of temporal modulation (usually PPM). 
In particular, in the case of a PPM/PAM TH signal, the temporal support $T_{n}$ is a regular $T$-spaced grid,

$$
T_{n}=U+n L_{c} T,
$$

where $U$ is a $\left[0, L_{c} T\right]$ uniformly distributed random variable. Figure 1 depicts a TH signal with a signature $\left\{c_{n}\right\}$ with period $L_{c}=3$.

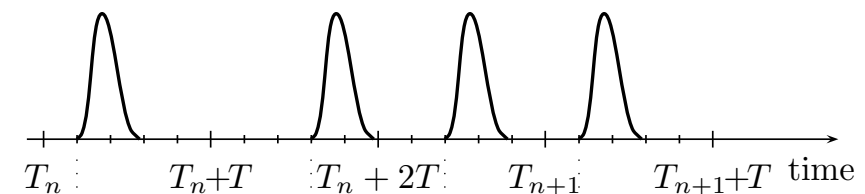
$T_{n}+c_{0} T_{c} \quad T_{n}+T+c_{1} T_{c} \quad T_{n}+2 T+c_{2} T_{c} T_{n+1}+c_{0} T_{c}$

Fig. 1. Time-hopping signal with signature $\left\{c_{0}, c_{1}, c_{2}\right\}$, where $T_{c}=T / 4, c_{0}=1, c_{1}=3$, and $c_{2}=3$.

A general model for $\mathrm{TH}$ and PPM/PAM signals in the presence of jitter and random losses is then given by equation (6), which corresponds to a shot noise with random excitation (1) with impulse response

$$
\begin{aligned}
h(t, Z) & := \\
& Z^{\mathrm{A}} \sum_{l=0}^{L_{c}-1} Z_{l}^{\mathrm{L}} w\left(t-Z^{\mathrm{P}}-l T-c_{l} T_{c}-Z_{l}^{\mathrm{J}}\right),
\end{aligned}
$$

where $\left\{Z_{n}^{\mathrm{A}}\right\}_{n \in \mathbb{Z}}$ and $\left\{Z_{n}^{\mathrm{P}}\right\}_{n \in \mathbb{Z}}$ models respectively pulse amplitude and position modulation, and the i.i.d. sequences of i.i.d. $L_{c}$-uplets

$$
\left\{\left(Z_{0 ; n}^{\mathrm{J}}, \ldots, Z_{L_{c}-1 ; n}^{\mathrm{J}}\right)\right\}_{n \in \mathbb{Z}},
$$

and

$$
\left\{\left(Z_{0 ; n}^{\mathrm{L}}, \ldots, Z_{L_{c}-1 ; n}^{\mathrm{L}}\right)\right\}_{n \in \mathbb{Z}},
$$

model, respectively, the random displacements and the random losses ( $Z^{\mathrm{L}}$ is a binary sequence). We remark that, due to the clustered nature of the random times, the introduction of $L_{c}$-uplets is necessary in order to consider jitter and thinning of each pulse of the signature.

Notice that expression (6) is an extension of the model presented in [2] that takes into account random losses of the pulses as well as a general jitter. Moreover, the shot noise approach we have adopted allows to easily complexify the model ad libitum. For instance, expression (6) can be easily extended to take also into account correlated amplitude modulation, correlated losses and pulse distortion (see [13], [17]).

\section{Direct-Sequence Time-Hopping Pulse Position and Amplitude Modulated Signals}

As for time-hopping, a direct-sequence signal allows for multi-user detection. It consists of multiplying a stream of pulses by a periodic deterministic sequence of +1 and -1 (the signature). It can be generally modeled as

$$
X(t)=\sum_{n \in \mathbb{N}} \sum_{k=0}^{L_{d}-1} a_{k}^{\mathrm{DS}} w\left(t-T_{n L_{d}+k}\right)
$$

where

- $L_{d}$ is the period of the signature sequence;

- $a_{k}^{\mathrm{DS}}, k=0, \ldots, L_{d}-1$, is the signature sequence, where $a^{\mathrm{DS}} \in\{+1,-1\}$.

Figure 2 depicts a direct-sequence signal.

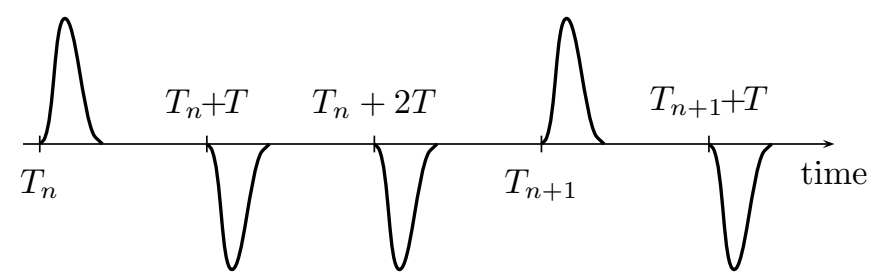

Fig. 2. Direct-sequence signal with signature $\left\{a_{0}, a_{1}, a_{2}\right\}$, where $a_{0}=1, a_{1}=-1$, and $a_{2}=-1$.

Modeling a direct-sequence time-hopping with PPM and PAM in the presence of jitter and random losses it is a matter of adding the feature "directsequence" to the model we have presented for the time-hopping with PPM and PAM. For notation ease, we consider that the direct-sequence and the timehopping signatures have the same length or period (i.e., $L_{d}=L_{c}$ ). We call $L$ such a common period (see [13] for the case $L_{d} \neq L_{c}$ ).

As previously done, we introduce the i.i.d. sequences of i.i.d. $L$-uplets $\left\{\left(Z_{0 ; n}^{\mathrm{J}}, \ldots, Z_{L-1 ; n}^{\mathrm{J}}\right)\right\}$ and $\left\{\left(Z_{0 ; n}^{\mathrm{L}}, \ldots, Z_{L-1 ; n}^{\mathrm{L}}\right)\right\}, n \in \mathbb{Z}$, to model, respectively, the random displacements and the random losses. Let $\left\{Z_{n}^{\text {P }}\right\}_{n \in \mathbb{Z}}$ be the i.i.d. sequence modeling the position modulation and $\left\{Z_{n}^{\mathrm{A}}\right\}_{n \in \mathbb{Z}}$ be the i.i.d. sequence modeling the amplitude modulation. A direct-sequence time-hopping with PPM and PAM signal in the presence of jitter and random losses then reads as in equation (9), where $a^{\text {DS }}$ represent the direct-sequence signature, $Z^{\mathrm{A}}$ models PAM random amplitudes, $Z^{\mathrm{L}}$ models the random losses, $Z^{\mathrm{P}}$ models PPM random positions and $Z^{\mathrm{J}}$ models the jitter. It corresponds to 


$$
\begin{gathered}
X(t)=\sum_{k=-\infty}^{\infty} Z_{n}^{\mathrm{A}} \sum_{l=0}^{L_{c}-1} Z_{l ; n}^{\mathrm{L}} w\left(t-U-n L_{c} T-Z_{n}^{\mathrm{P}}-l T-c_{l} T_{c}-Z_{l ; n}^{\mathrm{J}}\right) . \\
X(t)=\sum_{n \in \mathbb{N}} Z_{n}^{\mathrm{A}} \sum_{k=0}^{L_{d}-1} Z_{k ; n}^{\mathrm{L}} a_{k}^{\mathrm{DS}} w\left(t-U-n L T-Z_{n}^{\mathrm{P}}-k T-c_{k} T_{c}-Z_{k ; n}^{\mathrm{J}}\right) .
\end{gathered}
$$

a shot noise with random excitation (1) where we and have

$$
Z:=\left(Z^{\mathrm{A}}, Z^{\mathrm{P}},\left(Z_{0}^{\mathrm{J}}, \ldots, Z_{L-1}^{\mathrm{J}}\right),\left(Z_{0}^{\mathrm{L}}, \ldots, Z_{L-1}^{\mathrm{L}}\right)\right)
$$

$$
\begin{aligned}
\mathrm{E}\left[|\widehat{h}(\nu, Z)|^{2}\right]=|\widehat{w}(\nu)|^{2} \mathrm{E}\left[\left|Z^{\mathrm{A}}\right|^{2}\right] & {\left[L \mathrm{E}\left[\left|Z^{\mathrm{L}}\right|^{2}\right]+\left|\mathrm{E}\left[Z^{\mathrm{L}}\right]\right|^{2}\left|\phi_{Z^{\mathrm{s}}}(2 \pi \nu)\right|^{2}\right.} \\
& \left.\left(\left|\sum_{l=0}^{L-1} a_{l}^{\mathrm{DS}} e^{-i 2 \pi \nu\left(l T+c_{l} T_{c}\right)}\right|^{2}-L_{c}\right)\right] .
\end{aligned}
$$

$$
\begin{aligned}
& h(t, Z):= \\
& \quad Z^{\mathrm{A}} \sum_{k=0}^{L-1} Z_{k}^{\mathrm{L}} a_{k}^{\mathrm{DS}} w\left(t-Z^{\mathrm{P}}-k T-c_{k} T_{c}-Z_{k}^{\mathrm{J}}\right) .
\end{aligned}
$$

\section{Power Spectra}

The power spectral density of a shot noise with random excitation (1) is given by [13], [18]

$$
\begin{aligned}
S_{X}(\nu)=|\mathrm{E}[\widehat{h}(\nu, Z)]|^{2} S_{N}(\nu) & \\
& +\lambda \operatorname{Var}(\widehat{h}(\nu, Z)),
\end{aligned}
$$

where $\widehat{h}(\nu, z)$ is the Fourier transform of $h(t, z)$ (with respect to $t$ ) and $S_{N}(\nu)$ is the power spectral (pseudo) density of the sequence of random times $\left\{T_{n}\right\}_{n \in \mathbb{Z}}$. This general formula straightforwardly provides the spectrum of UWB signals.

We consider the spectrum of a direct-sequence time-hopping PPM/PAM signal with jitter and thinning, since the other configurations (single PPM and/or PAM with/without jitter and/or random losses) can be seen as particular cases.

From the expression of the impulse response (10), we obtain the expressions of is first order and second order moments,

$$
\begin{aligned}
& \mathrm{E}[\widehat{h}(\nu, Z)]=\widehat{w}(\nu) \mathrm{E}\left[Z^{\mathrm{A}}\right] \mathrm{E}\left[Z^{\mathrm{L}}\right] \\
& \phi_{Z^{\mathrm{P}}}(-2 \pi \nu) \phi_{Z^{\mathrm{J}}}(-2 \pi \nu) \sum_{k=0}^{L-1} a_{k}^{\mathrm{DS}} e^{-i 2 \pi \nu\left(k T+c_{k} T_{c}\right)},
\end{aligned}
$$

The power spectral (pseudo) density of the sequence of random times (5) is

$$
S_{N}(\nu)=\frac{1}{L T} \sum_{n \neq 0} \delta\left(\nu-\frac{n}{L T}\right)
$$

Finally, using equation (11), the power spectral density of a direct-sequence time-hopping PPM/PAM is given by

$$
\begin{gathered}
\mathcal{S}_{X}(\nu)=|\widehat{w}(\nu)|^{2} \frac{1}{L T} \\
\left(\left|\mathrm{E}\left[Z^{\mathrm{A}}\right]\right|^{2}\left|\mathrm{E}\left[Z^{\mathrm{L}}\right]\right|^{2}\left|\phi_{Z^{\mathrm{p}}}(2 \pi \nu)\right|^{2}\left|\phi_{Z^{\mathrm{s}}}(2 \pi \nu)\right|^{2}\right. \\
\left|\sum_{l=0}^{L-1} a_{l}^{\mathrm{DS}} e^{-i 2 \pi \nu\left(l T+c_{l} T_{c}\right)}\right|^{2} \sum_{n \neq 0} \delta\left(\nu-\frac{n}{L T}\right) \\
+L_{c} \mathrm{E}\left[\left|Z^{\mathrm{A}}\right|^{2}\right]\left(\mathrm{E}\left[\left|Z^{\mathrm{L}}\right|^{2}\right]-\left|\mathrm{E}\left[Z^{\mathrm{L}}\right]\right|^{2}\left|\phi_{Z^{\mathrm{s}}}(2 \pi \nu)\right|^{2}\right) \\
+\left|\mathrm{E}\left[Z^{\mathrm{L}}\right]\right|^{2}\left|\phi_{Z^{\mathrm{s}}}(2 \pi \nu)\right|^{2}\left|\sum_{l=0}^{L_{c}-1} a_{l}^{\mathrm{DS}} e^{-i 2 \pi \nu\left(l T+c_{l} T_{c}\right)}\right|^{2} \\
\left.\left(\mathrm{E}\left[\left|Z^{\mathrm{A}}\right|^{2}\right]-\left|\mathrm{E}\left[Z^{\mathrm{A}}\right]\right|^{2}\left|\phi_{Z^{\mathrm{P}}}(2 \pi \nu)\right|^{2}\right)\right) .
\end{gathered}
$$

where $\widehat{w}(\nu)$ is the Fourier transform of the pulse shape, $\phi_{Z^{\mathrm{p}}}(u)$ and $\phi_{Z^{\mathrm{s}}}(u)$ are the characteristic functions of the random displacements due to the position modulation and of the random displacements due to the clock jitter, respectively. 
We remark that in the above spectral formula each feature of the model (pulse shape, amplitude modulation, position modulation, time-hopping signature, random losses and clock jitter) has a corresponding spectral term that appears separately and explicitly: this is a key property for model analysis. Moreover, additional features (random pulse distortion for instance) can be straightforwardly taken into account by mean of the vector of random parameters $Z$ still providing explicit expressions of the power spectra.

\section{Conclusions}

Shot noises are a natural language for modeling the large family of UWB signals. Their spectral formulas provide a perfect toolbox for evaluating the spectra of UWB signals. They unify well known results and provide spectral expressions for highly complex UWB signals. Moreover, the corresponding approach is modular allowing to build progressively specific features of the model in a systematic manner. In addition the exact power spectrum expression we have derived are easy to understand since the contribution of the various features of the model appears explicitly and separately: such a peculiarity of our approach has a tremendous impact in the design and the analysis of UWB system. Spectrum of direct-sequence TH PPM/PAM signals is, as far as we know, an original contribution. We emphasize the generality of the result that presents a generic directsequence and $\mathrm{TH}$, a generic jitter and generic losses. Moreover, different pulse modulations can be taken into account by simply replacing the spectrum $\mathcal{S}_{N}$ and the random pulse function.

\section{REFERENCES}

[1] M. Win and R. Scholtz, "Impulse radio: how it works," IEEE Communications Letters, vol. 2, no. 2, pp. 36-38, February 1998.

[2] M. Win, "A unified spectral analysis of generalized timehopping spread-spectrum signals in the presence of timing jitter," IEEE J. Select. Areas Commun., vol. 20, no. 9, pp. 1664-1676, December 2002.
[3] J. Romme and L. Piazzo, "On the power spectral density of time-hopping impulse radio," in IEEE Conference on Ultra Wideband Systems and Technologies, 2002, pp. 241-244.

[4] P. Brémaud and A. Ridolfi, "Power spectra of uwb timehopping modulated signals: a shot noise approach," in IEEE ISIT, 2004.

[5] G. Cariolaro, T. Erseghe, and L. Vangelista, "Exact spectral evaluation of the family of digital pulse interval modulated signals," IEEE Trans. Inform. Theory, vol. 47, no. 7, pp. 2983-2992, November 2001.

[6] L. Bondesson, "Shot-noise processes and shot-noise distributions," in Encyclopedia of Statistical Sciences, N. L. J. . S. Kotz, Ed. Wiley, New York, 1988, vol. 8, pp. 448-452.

[7] J. A. Gubner, "Computation of shot-noise probability distributions and densities," SIAM J. Sci. Comput., vol. 17, no. 3, pp. 750-761, 1996.

[8] M. Parulekar and A. M. Makowski, "M/G/ $\infty$ input processes: A versatile class of models for network traffic," in Proceeding of INFOCOM '97, vol. 2. IEEE Computer and Communications Societies, 1997, pp. 419-426.

[9] D. Snyder and C. Georghiades, "Design of coding and modulation for power-efficient use of a band-limited optical channel," IEEE Trans. Comm., vol. 31, no. 4, pp. 560-565, 1980

[10] C. Georghiades, "Some optimal and suboptimal receivers deriving information from nonsynchronized poisson data," IEEE Trans. Inform. Theory, vol. 35, no. 1, pp. 157-165, 1989.

[11] R. J. Fontana, "An insight into uwb interference from a shot noise perspective," in IEEE Conference on Ultra Wideband Systems and Technologies, 2002, pp. 309- 313.

[12] M. Win and R. Scholtz, "Ultra-wide bandwidth timehopping spread-spectrum impulse radio for wireless multiple-access communications," IEEE Trans. Comm., vol. 48, no. 4, pp. 679-689, April 2000.

[13] A. Ridolfi, "Power spectra of random spikes and related complex signals, with application to communications," Ph.D. dissertation, Ecole Politechnique Fédérale de Lausanne - EPFL, 2004.

[14] D. Middleton, Statistical Communication Theory. McGraw-Hill, 1960.

[15] M. Schwartz, W. R. Bennet, and S. Stein, Communication Systems and Techniques. McGraw-Hill, 1966.

[16] K. Siwiak, "Ultra-wide band radio: introducing a new technology," in IEEE Vehicular Technology Conference, vol. 2, 2001, pp. 1088-1093.

[17] P. Brémaud and A. Ridolfi, "Power spectra related to UWB communications," in IEEE ICC, 2004.

[18] P. Brémaud, L. Massoulié, and A. Ridolfi, "Power spectra of random spike fields and related processes," Journal of Applied Probability, 2005. 